\title{
Bosonized noncommutative bi-fundamental fermion and S-duality
}

\author{
Harold Blas \\ Departamento de Matemática - ICET \\ Universidade Federal de Mato Grosso \\ Av. Fernando Correa, s/n, Coxipó \\ 78060-900, Cuiabá - MT - Brazil
}

\begin{abstract}
We perform the path-integral bosonization of the recently proposed noncommutative massive Thirring model $\left(\mathrm{NCMT}_{1}\right)$ [JHEP0503(2005)037]. This model presents two types of current-current interaction terms related to the bi-fundamental representation of the group $U(1)$. Firstly, we address the bosonization of a bi-fundamental free Dirac fermion defined on a noncommutative (NC) Euclidean plane $\mathbb{R}_{\theta}^{2}$. In this case we show that the fermion system is dual to two copies of the NC Wess-Zumino-Novikov-Witten model. Next, we apply the bosonization prescription to the $\mathrm{NCMT}_{1}$ model living on $\mathbb{R}_{\theta}^{2}$ and show that this model is equivalent to two-copies of the WZNW model and a twofield potential defined for scalar fields corresponding to the global $U(1) \times U(1)$ symmetry plus additional bosonized terms for the four fermion interactions. The bosonic sector resembles to the one proposed by Lechtenfeld et al. [Nucl. Phys. B705(2005)477] as the noncommutative sine-Gordon for a pair of scalar fields. The bosonic and fermionic couplings are related by a strong-weak duality. We show that the couplings of the both sectors for some representations satisfy similar relationships up to relevant re-scalings, thus the NC bi-fundamental couplings are two times the corresponding ones of the NC fundamental (anti-fundamental) and eight times the couplings of the ordinary massive Thirring and sine-Gordon models.
\end{abstract}




\section{Introduction}

Quantum field theories on non-commutative (NC) space-times are receiving considerable attention in recent years in connection to string and $M$ theories (see, Refs. [1]). In the case of open string with $N=2$ world-sheet super-symmetries and for target space filled by $n$ coincident $D 3$-branes the low-energy NC model corresponds to NC self-dual $U(n)$ Yang Mills theory (NC SDYM) [2]. This theory is integrable classically [3] and possesses a factorized S-matrix in the quantum version [2]. It is a remarkable fact that almost all NC integrable models in less than four dimensions can be obtained by dimensional reduction of four dimensional NC SDYM (see e.g. [4] and references therein).

Some non-commutative versions of the sine-Gordon $\left(\mathrm{NCSG}_{1,2}\right)$ [4]-[8] and corresponding massive Thirring models $\left(\mathrm{NCMT}_{1,2}\right)$ [8] have been proposed in the literature. Their relevant $\mathrm{NC}$ equations of motion have the general property of reproducing the ordinary models in the commutative limit. The Grisaru-Penati version $\mathrm{NCSG}_{2}[5,6]$ introduces a constraint which is non-trivial only in the non-commutative case. However, at the quantum level this model gives rise to particle production as was discovered by evaluating tree-level scattering amplitudes [6]. Moreover, introducing an extra field, Lechtenfeld et al. [4] proposed a novel $\mathrm{NCSG}_{1}$ model which presents a factorizable and causal S-matrix at tree level computations. It seems to be that requiring an infinite number of classical conserved quantities does not uniquely reproduce the Moyal deformation of two-dimensional integrable field theories. The models $\mathrm{NCSG}_{1,2}$ were recently derived from the NC self-dual $U(2)$ Yang Mills theory through dimensional and algebraic reduction processes [6, 4]. Moreover, these scalar field models $\left(\mathrm{NCSG}_{1,2}\right)$ were shown to be related to spinorial models on the classical level, possessing soliton solutions in both sectors and a strong-weak mapping of their relevant coupling constants. These models, respectively named as $\mathrm{NCMT}_{1,2}$, are Moyal extensions of the usual massive Thirring model (MT) defined for a bi-fundamental $(b f)$ fermion with two types of current-current interaction terms related to the group $U(1) \times U(1)$ [8]. The $\mathrm{NCMT}_{2}$ model requires two copies of the $\mathrm{NCMT}_{1}$ theory, this last model being just the $\mathrm{NC}$ extension of the massive Thirring for a bi-fundamental fermion.

On the other hand, the equivalence of the ordinary sine-Gordon and massive Thirring models is a two-dimensional example of having different field representations of the same theory. The massive Thirring model (MT)

$$
\mathcal{L}_{M T}=i \bar{\psi} \gamma^{\mu} \partial_{\mu} \psi+m \bar{\psi} \psi-\frac{\lambda_{M T}}{2} j^{\mu} j_{\mu}
$$

is equivalent to the sine-Gordon model (SG) [9]

$$
\mathcal{L}_{S G}=\frac{1}{2} \partial_{\mu} \phi \partial^{\mu} \phi+\frac{\alpha_{0}}{\beta_{S G}^{2}}\left(\cos \beta_{S G} \phi-1\right) .
$$

This duality is expressed in terms of the well-known abelian bosonization rules. The two field representations (1.1) and (1.2) describing the same physics are useful in different coupling regimes which are related by

$$
\frac{4 \pi}{\beta_{S G}^{2}}=1+\frac{\lambda_{M T}}{\pi}
$$


The particle/soliton correspondence in the context of this duality has been uncovered through the master Lagrangian and symplectic quantization approaches [10] of the higher grading $\operatorname{sl}(2)$ affine Toda model coupled to matter (Dirac) field [11]. A generalization for any (untwisted) affine Lie algebra has been provided in [12].

Here we consider the problem of extending this duality to the NC spacetime using the path-integral formalism. This program, initiated in [13], considers the abelian bosonization of a free fermion in two noncommuting dimensions $[14,15]$ using path-integral techniques developed in refs. [16]-[19]. We point out that in these NC treatments the Dirac fermion was assumed to be in the anti-fundamental $(\bar{f})$ representation of the $U(1)$ group. Next, we summarize the main features that emerged from this bosonization procedure. The free fermion action is bosonized to a $\mathrm{NC} U(1)$ WZW-action in which the WZ term in the action gives a non-trivial contribution due to the non-commutativity of spacetime. The procedure resembles closely the conventional non-abelian bosonization [20] with the bosonization rules being similar both in the non-abelian and NC cases. The massive Thirring model with antifundamental fermion is dual to a WZW model plus a NC cosine potential and an additional quartic term emerging from the $\mathrm{NC}$ bosonization of the current-current interaction. In this way, except for the quartic term, a copy of the Grisaru-Penati model emerges in the bosonic sector [13]. Recall that the Grisaru-Penati model $[5,6,8]$ is defined by the sum of a WZNW action for $e_{\star}^{i \beta_{\bar{f}} \phi} \in U(1)_{C}$ and a cosine potential $\left(e_{\star}^{i \beta_{\bar{f}} \phi}+e_{\star}^{-i \beta_{\bar{f}} \phi}\right)$, plus some copies of these terms written for $e_{\star}^{i \beta_{\bar{f}} \phi^{\dagger}}, \beta_{\bar{f}}$ being the bosonic coupling constant. The same type of duality relationship, eq. (1.3), emerges up to couplings re-scalings [13]

$$
\frac{16 \pi}{\beta_{\bar{f}}^{2}}=1+\frac{\lambda_{\bar{f}}}{4 \pi},
$$

thus realizing an example of S-duality in NC spacetime. The S-duality in the context of NC geometry has also been discussed in Refs. [21].

The Grisaru-Penati model, in spite of integrability in the sense that it possesses an infinite number of conserved quantities, features an acausal S-matrix and particle production takes place. It was recently shown, on the classical level, that this model corresponds to the Moyal product extension of two copies of the ordinary massive Thirring model written for a bifundamental fermion [8]. The main features observed in this classical correspondence, apart from the doubling in the number of fermion fields, is that, even though the strong-weak duality in the soliton sector is preserved, only the unitary subgroup of the $U(1)_{C} \times U(1)_{C}$ symmetry of the bosonic model is realized in the spinorial model.

In two-dimensional Minkowski spacetime, a Moyal deformation necessarily implies the noncommutativity of the time coordinate and the causality and unitarity properties of the theory can be spoiled [22]. However, in an exactly solvable quantum field theory this feature can be improved as has been observed in the NC Lee model, where it was shown that the model is free from the $I R / U V$ mixing in both the space-space and space-time noncommutative cases. Due to the absence of this type of mixing one can expect that the theory is unitary [23].

In this context, it would be interesting to understand what actually determines the systems to be integrable (in the sense of possessing a factorizable and causal S-matrix) and 
dual to each other. From the considerations summarized above on NC extensions of SG model, the correspondence $\mathrm{NCSG}_{1} \rightarrow \mathrm{NCMT}_{1}$ is promising and deserves a further investigation. The main features of the $\mathrm{NCSG}_{1}$ model are the presence of multi-soliton solutions, the factorizable and causal S-matrix (verified at tree level) despite of the space-time noncommutativity [4]. Moreover, these models originate directly through reduction processes starting from the NC WZNW type action for the $G L(2)$ affine Toda field coupled to the higher grading matter $\left(\right.$ Dirac) fields $\left(\mathrm{NCATM}_{1}\right)$, such that the $U(1) \times U(1)$ symmetry is relevant, both in the construction of the $\mathrm{NCSG}_{1}$ model and in the star-localized Noether procedure to construct the $U(1) \times U(1)$ currents in the $\mathrm{NCMT}_{1}$ sector [8]. Then, it is interesting to perform the bosonization of the massive Thirring theory $\left(\mathrm{NCMT}_{1}\right)$ as defined in [8] and study the corresponding bosonic theory properties; e.g. to answer the question of what features it shares with the classical $\mathrm{NCSG}_{1}$ theory.

The paper is organized as follows. In the next section we perform the $\mathrm{NC}$ bosonization of the bi-fundamental free fermion. In section 3 we bosonize the $\mathrm{NCMT}_{1}$ theory making use of the bosonization rules for the $\mathrm{NC}$ free fermion. In subsection 3.1 we bosonize the currentcurrent interaction terms and identify the canonical bosonic physical field, related to the global $U(1)$ charge, coming from the kinetic terms and from the boson quartic interactions. In subsection 3.2 we bosonize the mass term by axial symmetry consideration alone and get a two-field potential. Next, considering the physical field and the relevant coupling constants we establish the S-duality for the NC field theories under consideration. Moreover, we record the bosonized model in matrix form which is of the type proposed by Lechtenfeld et al. $\left(\mathrm{NCSG}_{1}\right)$ except for the quartic contributions coming from the current-current interactions. Finally, the conclusions and discussions are presented in section 4 .

\section{The bi-fundamental fermion and NC bosonization}

A conventional system of $N$ free massless Dirac fermions is equivalent to a bosonic theory governed by the WZW model for a bosonic field $g \in S U(N)$ and a real bosonic field $\phi$ with a free scalar action [20]. On the other hand, the massless free fermion in the fundamental representation or anti-fundamental representation $[14,15,13]$ of the abelian $U(1)$ group on

$\mathbb{R}_{\theta}^{2}$ is equivalent to a noncommutative version of the WZW model $[14,24]$. In this section we derive the bosonization rules for the bi-fundamental free fermion action defined on $\mathbb{R}_{\theta}^{2}$. We follow the path integral approach developed in $[18,19,13]$.

We consider the bi-fundamental $U(1) \times U(1)$ free fermion theory

$$
S_{b f}=\int d^{2} x i \bar{\psi}_{i_{1}}^{j_{2}} \delta_{i_{2}}^{i_{1}} \delta_{j_{2}}^{j_{1}} \star \not \partial \psi_{j_{1}}^{i_{2}}
$$

where the group indices $i$ corresponds to the first $U(1)$ and the indices $j$ to the second $U(1)$. We will see that this theory is equivalent to a bosonic model by showing that the correlation functions constructed from the both theories are equal. So, let us write the generating functional for the correlations as

$$
Z\left[s_{1}, s_{2}\right]=\int \mathcal{D} \bar{\psi} \mathcal{D} \psi \exp \left\{-\int d^{2} x \bar{\psi}_{i_{1}}^{j_{1}} \star\left(i \not \supset\left[s^{(1)}, s^{(2)}\right] \psi\right)_{j_{1}}^{i_{1}}\right\}
$$


where

$$
\left\{D_{\mu}\left[s^{(1)}, s^{(2)}\right] \psi(x)\right\}_{j_{1}}^{i_{1}} \equiv\left[\partial_{\mu} \delta_{i_{2}}^{i_{1}} \delta_{j_{1}}^{j_{2}}+i \star \delta_{i_{2}}^{i_{1}} s_{\mu j_{1}}^{(1) j_{2}}-i \delta_{j_{1}}^{j_{2}} s_{\mu i_{2}}^{(2) i_{1}} \star\right] \psi(x)_{j_{2}}^{i_{2}},
$$

with the notation $\left[\star s_{\mu}^{(1)}(x)\right] \psi(x) \equiv \psi(x) \star s_{\mu}^{(1)}(x)$ and $\left[s_{\mu}^{(2)}(x) \star\right] \psi(x) \equiv s_{\mu}^{(2)}(x) \star \psi(x)$.

Notice that in the above generating functional we have considered two types of external sources, i.e. $s_{\mu}^{(1)}$ and $s_{\mu}^{(2)}$, such that differentiations with respect to each source provide correlation functions between the relevant currents

$$
\begin{aligned}
j^{(1) \mu} & \equiv \bar{\psi} \star \gamma^{\mu} \psi ; \\
j^{(2) \mu} & \equiv \psi_{\beta}\left(\gamma^{\mu}\right)_{\alpha \beta} \star \bar{\psi}_{\alpha},
\end{aligned}
$$

respectively.

The currents $j^{(1) \mu}$ and $j^{(2) \mu}$ as defined above differ only by a sign in the commutative limit, whereas on NC Euclidean space they are different [25]. Next, we briefly describe the steps followed to obtain the currents (2.4)-(2.5) associated to a bi-fundamental fermion system. In order to obtain the currents by the Noether procedure we make the global transformation localized, as discussed in [26] the star-localized procedure is not unique in the $\mathrm{NC}$ case. In fact, the most general $U(1) \mathrm{x} U(1)$ symmetry in $\mathrm{NC}$ space given for a charged bi-fundamental field is provided by the transformation rules $[26,27]$

$$
\psi \rightarrow u_{2}(x) \star \psi \star u_{1}^{-1}(x) ; \quad \bar{\psi} \rightarrow u_{1}(x) \star \bar{\psi} \star u_{2}^{-1}(x), \quad u_{1}(x)=e_{\star}^{-i \alpha_{1}(x)}, u_{2}(x)=e_{\star}^{i \alpha_{2}(x)},
$$

where $u_{1,2}(x)$ are independent starred exponentials with $\alpha_{1,2}(x)=$ real functions. The two currents above (2.4)-(2.5) correspond to the symmetries $u_{1}$ and $u_{2}$, respectively.

Since the charge is associated to global transformation of the charged field for which there is no difference between the ordinary and non-commutative product, one can conclude that the currents $j^{(1) \mu}$ and $j^{(2) \mu}$ share the same charge. In fact, for global $u_{1,2}$ only the product

$$
u_{\text {global }}=u_{2} u_{1}^{-1}
$$

is relevant.

In the following we use the approach developed in $[15,13]$ conveniently adapted to our problem. The measure in (2.2) is invariant under the local transformations of the fermion fields (2.6) and consequently the generating functional (2.2) is gauge invariant under separate gauge transformations acting on $s^{(1)}$ and $s^{(2)}$, namely

$$
Z\left[s^{(1)}, s^{(2)}\right]=Z\left[s^{(1) U_{1}}, s^{(2) U_{2}}\right]
$$

provided that the sources transform according to

$$
s_{\mu}^{(n)} \rightarrow s_{\mu}^{(n) U_{n}}=U_{n} \star s_{\mu}^{(n)} \star U_{n}^{-1}+U_{n} \partial_{\mu} U_{n}^{-1}, \quad n=1,2 .
$$

Then from the identity (2.8) one gets

$$
\begin{aligned}
Z\left[s^{(1)}, s^{(2)}\right] & =\int \mathcal{D} \bar{\psi} \mathcal{D} \psi \mathcal{D} U_{1} \mathcal{D} U_{2} \exp \left\{-\int d^{2} x \bar{\psi}\left(i \star \not \partial-\star \phi^{(1) U_{1}}+\xi^{(2) U_{2}} \star\right) \psi\right\} \\
& =\int \mathcal{D} U_{1} \mathcal{D} U_{2} \operatorname{det}\left(i \not \partial-\star \phi^{(1) U_{1}}+\phi^{(2)} U_{2} \star\right),
\end{aligned}
$$


where the fermions have been integrated out. Notice that the integration over the $U_{n}$ 's just amounts to a change of the overall normalization.

It is useful to introduce an explicit representation of the group $g \in U(1) \times U(1)$ in the form

$$
g=\left(\begin{array}{cc}
e_{\star}^{i \Lambda_{2}} & 0 \\
0 & e_{\star}^{-i \Lambda_{1}}
\end{array}\right) \equiv g_{2} \star g_{1}=g_{1} \star g_{2}, \quad g_{2}=\left(\begin{array}{cc}
e_{\star}^{i \Lambda_{2}} & 0 \\
0 & 1
\end{array}\right), \quad g_{1}=\left(\begin{array}{cc}
1 & 0 \\
0 & e_{\star}^{-i \Lambda_{1}}
\end{array}\right),
$$

where $\Lambda_{1,2}$ are real fields.

In this $2 \times 2$ representation the matrix Lie algebra valued fields $\phi^{(n)}(n=1,2)$ corresponding to each subalgebra respectively has just one entry different from zero, respectively. Then the matrix of interest becomes

$$
\begin{aligned}
\left(i \star \not \partial-\star \phi^{(1) U_{1}}+\phi^{(2) U_{2}} \star\right)_{2 \times 2} & =\left(\begin{array}{cc}
i \not \partial+\phi^{(2) U_{2} \star} & 0 \\
0 & i \not \partial-\star \phi^{(1)} U_{1}
\end{array}\right) \\
& =\left(\begin{array}{cc}
i \not \partial+\phi^{(2) U_{2} \star} & 0 \\
0 & 1
\end{array}\right)\left(\begin{array}{cc}
1 & 0 \\
0 & i \not \partial-\star \phi^{(1)} U_{1}
\end{array}\right),
\end{aligned}
$$

where in the right hand sides of $(2.12)$ the fields $s^{(n) U_{n}}(n=1,2)$ denote just functions instead of Lie algebra valued fields.

Therefore the generator functional factorizes

$$
Z\left[s^{(1)}, s^{(2)}\right]=\int \mathcal{D} U_{1} \mathcal{D} U_{2} \operatorname{det}\left(i \not \partial-\star \phi^{(1) U_{1}}\right) \operatorname{det}\left(i \not \partial+\phi^{(2) U_{2}} \star\right),
$$

where the Lie algebra valued character has been restored for the fields $\xi^{(n)} U_{n}$.

This factorization is expected since $Z\left[s^{(1)}, s^{(2)}\right]$ is gauge invariant under the separate gauge transformations $(2.9)$ acting on $s^{(1)}$ and $s^{(2)}$, respectively

Let us introduce the connections through

$$
b_{\mu}^{(n)}=s_{\mu}^{(n) U_{n}}
$$

such that the field strengths of $b_{\mu}^{(n)}$ and $s_{\mu}^{(n)}$ satisfy

$$
F_{\mu \nu}^{(n)}\left[b^{(n)}\right]=U_{n} \star F_{\mu \nu}^{(n)}\left[s^{(n)}\right] \star U_{n}^{-1}, \quad n=1,2 .
$$

We proceed by changing the $U_{n}$ integrations to integrations over the new connections $b^{(n)}$. In order to achieve that we shall use the identity [19]

$$
\int \mathcal{D} b_{\mu}^{(n)} \mathcal{P}\left[b_{\mu}^{(n)}\right] \delta\left[\epsilon^{\mu \nu}\left(F_{\mu \nu}^{(n)}\left[b^{(n)}\right]-F_{\mu \nu}^{(n)}\left[s^{(n)}\right]\right)\right]=\int \mathcal{D} U_{n} \mathcal{P}\left[s_{\mu}^{(n) U_{n}}\right], \quad \text { for } n=1,2 .
$$

where $\mathcal{P}$ is a gauge invariant function.

Making use of the identities (2.16) the generating functional (2.13) can be written as

$$
\begin{array}{r}
Z\left[s^{(1)}, s^{(2)}\right]=\int \mathcal{D} b_{\mu}^{(n)} \Delta_{n} \delta\left(b_{+}^{(n)}-s_{+}^{(n)}\right) \delta\left[\epsilon^{\mu \nu}\left(F_{\mu \nu}^{(n)}\left[b^{(n)}\right]-F_{\mu \nu}^{(n)}\left[s^{(n)}\right]\right)\right] \\
\times \operatorname{det}\left(i \not \partial-\star \not b^{(1)}\right) \operatorname{det}\left(i \not \partial+\not b^{(2)} \star\right),
\end{array}
$$


where we have chosen the gauge conditions $b_{+}^{(n)}=s_{+}^{(n)}, n=1,2$; with the $\Delta_{n}$ 's being the relevant Faddeev-Popov determinants.

We define the Lie algebra valued fields $\hat{a}_{n}$ and use them in order to reinforce the delta function conditions, i.e.

$$
\begin{aligned}
Z\left[s^{(n)}\right]= & \int \mathcal{D} \hat{a}_{n} \mathcal{D} b_{\mu}^{(n)} \Delta_{n} \delta\left(b_{+}^{(n)}-s_{+}^{(n)}\right) \operatorname{det}\left(i \not \partial-\star \not b^{(1)}\right) \operatorname{det}\left(i \not \partial+\not b^{(2)} \star\right) \\
& \times \Pi_{n} \exp \left(\mathcal{A}_{n}\right)
\end{aligned}
$$

where $\mathcal{A}_{n} \equiv-\frac{C_{n}}{8 \pi} \operatorname{tr} \int d^{2} x \hat{a}_{n} \delta\left[\epsilon^{\mu \nu}\left(F_{\mu \nu}^{(n)}\left[b^{(n)}\right]-F_{\mu \nu}^{(n)}\left[s^{(n)}\right]\right)\right]$, with the $C_{n}$ 's being some constants to be conveniently chosen below. In two dimensions one can parameterize a gauge boson as a pure gauge field, so the source fields $s_{\mu}^{(n)}$ and $b_{\mu}^{(n)}$ can be written in terms of group-valued variables

$$
\begin{aligned}
s_{+}^{(n)} & =i \widetilde{s}_{n}^{-1} \partial_{+} \widetilde{s}_{n} \\
s_{-}^{(n)} & =i s_{n} \partial_{-} s_{n}^{-1} \\
b_{+}^{(n)} & =i\left(\widetilde{b}_{n} \widetilde{s}_{n}\right)^{-1} \partial_{+}\left(\widetilde{b}_{n} \widetilde{s}_{n}\right) \\
b_{-}^{(n)} & =i\left(s_{n} b_{n}\right) \partial_{-}\left(s_{n} b_{n}\right)^{-1}
\end{aligned}
$$

The $U(1) \times U(1)$ group valued fields $s_{n}, \widetilde{s}_{n}, b_{n}, \widetilde{b}_{n}$ are in the same representation as the $g_{n}$ 's $(n=1,2)$ in $(2.11)$.

Notice that the determinant depending on $\not_{2}$ is associated to the fundamental fermion representation and the one with $\not b_{1}$ resembles to that of the anti-fundamental. The fundamental and anti-fundamental determinants coincide, as discussed in [15]. The exact effective action in each case is obtained by integrating the chiral anomaly $[14,15]$. On the other hand, in two dimensions the bi-fundamental Dirac fermion carries no mixed anomaly [28, 29] (for chiral matter see [30]). Therefore, one can make the affective action computation separately for each $U(1)$ sector. Taking into account (2.13) the fermion determinant for the parameterizations (2.19)-(2.22) can be written as $[14,15]$

$$
\operatorname{det}\left(i \not \partial-\star \not^{(1)}+\not b^{(2)} \star\right)=\exp \left(W\left[\widetilde{b}_{1} \star \widetilde{s}_{1} \star s_{1} \star b_{1}\right]\right) \exp \left(W\left[\widetilde{b}_{2} \star \widetilde{s}_{2} \star s_{2} \star b_{2}\right]\right),
$$

where

$$
W[g]=\frac{1}{8 \pi} \int_{\Sigma} d^{2} x \operatorname{Tr}\left(\partial_{\mu} g \star \partial_{\mu} g^{-1}\right)-\frac{i}{12 \pi} \int_{B} d^{3} x \epsilon^{i j k} \operatorname{Tr}\left(g^{-1} \star \partial_{i} g \star g^{-1} \star \partial_{j} g \star g^{-1} \star \partial_{i} g\right)(2
$$

is the NC extension of the WZNW model $[14,24]$. The manifold $\Sigma$, parameterized by $\left(x^{0}, x^{1}\right)$, is the boundary of the three-dimensional manifold $B$. The extra dimension $x^{2}$ is taken to be commutative.

The Jacobians for the change of variables from $b_{\mu}^{(n)}$ to $b_{n}, \widetilde{b}_{n}$ become [13]

$$
\begin{aligned}
\mathcal{D} b_{+}^{(n)} \mathcal{D} b_{-}^{(n)} & =\operatorname{det}_{\star} D_{+}\left[\widetilde{b}_{n} \star \widetilde{s}_{n}\right] \operatorname{det}_{\star} D_{-}[s \star b] \mathcal{D} \widetilde{b}_{n} \mathcal{D} b_{n} \\
& =\mathcal{D} \widetilde{b}_{n} \mathcal{D} b_{n} \exp \left(\kappa_{n} W\left[\widetilde{b}_{n} \star \widetilde{s}_{n} \star s_{n} \star b_{n}\right]\right), \quad n=1,2 ;
\end{aligned}
$$


where $\kappa_{n}$ is the parameter depending on the representation of the covariant derivative involved in the change of variables, and since we shall not use it here we leave it as an unspecified parameter.

The delta functional in (2.18), for these change of variables, can be written as

$$
\delta\left(b_{+}^{(n)}-s_{+}^{(n)}\right)=\frac{1}{\operatorname{det}_{\star} D_{+}\left[s_{+}^{(n)}\right]} \delta\left(\widetilde{b}^{(n)}-I\right) .
$$

Then, from the results above, (2.18) can be written as

$$
Z\left[s^{(n)}\right]=\int \mathcal{D} \hat{a}_{n} \mathcal{D} b_{n} \Pi_{n} \exp \left(\mathcal{B}_{n}\right) \exp \left(\left(1+\kappa_{n}\right) W\left[\widetilde{s}_{n} \star s_{n} \star b_{n}\right]\right)
$$

where

$$
\mathcal{B}_{n}=i \frac{C_{n}}{4 \pi} \operatorname{tr} \int d^{2} x\left(D_{+}\left[\widetilde{s}_{n}\right] \star \hat{a}_{n}\right) s_{n} \star b_{n} \star\left(\partial_{-} b_{n}^{-1}\right) \star s_{n}^{-1},
$$

and the covariant derivative is in the adjoint representation of the corresponding $U(1)$ group.

In the present approach to bosonization one introduces two group-valued fields, each one related to the corresponding $U(1)$ group, say $g_{1}$ and $g_{2}$, which will play the role of the boson fields equivalent to the original bi-fundamental fermionic model and will be invariant under gauge transformations. So, let us introduce a change of variables from the algebra-valued fields $\hat{a}_{n}$ to the group-valued fields $a_{n}$ as

$$
D_{+}\left[\widetilde{s}_{n}\right] \star \hat{a}_{n}=\widetilde{s}_{n}^{-1} \star\left(a_{n}^{-1} \star \partial_{+} a_{n} \star \widetilde{s}\right) .
$$

The Jacobian of these transformations are $[18,13]$

$$
\mathcal{D} \hat{a}_{n}=J_{L}^{(n)} \mathcal{D} a_{n}, \quad J_{L}^{(n)}=\exp \left(\kappa_{n} W\left[a_{n} \star \widetilde{s}_{n} \star s_{n}\right]-\kappa_{n} W\left[\widetilde{s}_{n} \star s_{n}\right]\right), \quad n=1,2 .
$$

Therefore, the generating functional becomes

$$
\begin{aligned}
Z\left[s^{(n)}\right]= & \int \mathcal{D} a_{n} \mathcal{D} b_{n} \Pi_{n} \exp \left(\left(1+\kappa_{n}\right) W\left[\widetilde{s}_{n} \star s_{n} \star b_{n}\right]+\kappa_{n} W\left[a_{n} \star \widetilde{s}_{n} \star s_{n}\right]-\kappa_{n} W\left[\widetilde{s}_{n} \star s_{n}\right]-\right. \\
& \left.\frac{C_{n}}{4 \pi} \operatorname{tr} \int d^{2} x \widetilde{s}_{n}^{-1} \star\left(a_{n}^{-1} \star \partial_{+} a_{n}\right) \star \widetilde{s}_{n} \star s_{n} \star\left(b_{n} \star \partial_{-} b_{n}^{-1}\right) \star s_{n}^{-1}\right) .
\end{aligned}
$$

Choosing the up to now arbitrary constants $C_{n}$ to be $C_{n} \equiv\left(1+\kappa_{n}\right)$ and making use of the Polyakov-Wiegmnann identity

$$
W\left[g_{2} \star g_{1}\right]=W\left[g_{2}\right]+W\left[g_{1}\right]-\frac{1}{4 \pi} \int_{\Sigma} d^{2} x \operatorname{Tr}\left(g_{2}^{-1} \star \partial_{+} g_{2} \star g_{1} \star \partial_{-} g_{1}^{-1}\right),
$$

one can write

$$
Z\left[s^{(n)}\right]=\int \mathcal{D} a_{n} \mathcal{D} b_{n} \Pi_{n} \exp \left(\left(1+\kappa_{n}\right) W\left[a_{n} \widetilde{s}_{n} s_{n} b_{n}\right]+W\left[\widetilde{s}_{n} s_{n}\right]-W\left[a_{n} \widetilde{s}_{n} s_{n}\right]\right)
$$

Since the integrations over the $b_{n}$ 's can be trivially factorized by making the changes $b_{n} \rightarrow \hat{b}_{n}=a_{n} \widetilde{s}_{n} s_{n} b_{n}$, each one with trivial Jacobian, one has

$$
Z\left[s^{(n)}\right]=\int \mathcal{D} a_{n} \Pi_{n} \exp \left(W\left[\widetilde{s}_{n} s_{n}\right]-W\left[a_{n} \widetilde{s}_{n} s_{n}\right]\right) .
$$


The final transformations $a_{n} \widetilde{s}_{n} s_{n} \rightarrow \widetilde{s}_{n} a_{n} s_{n}$ with trivial Jacobians; together with the Polyakov-Wiegmann identity provide us the boson counterpart of the bi-fundamental fermion model in (2.2) (as usual rename $a_{n}$ as $g_{n}$ )

$$
\begin{aligned}
Z\left[s_{ \pm}^{(n)}\right]= & \int \Pi_{n} \mathcal{D} g_{n} \exp \left[-W\left[g_{n}\right]+\frac{i}{4 \pi} \operatorname{tr} \int d^{2} x\left(s_{+}^{(n)} \star g_{n} \star \partial_{-} g_{n}^{-1}+s_{-}^{(n)} \star g_{n}^{-1} \star \partial_{+} g_{n}\right)+\right. \\
& \left.\frac{1}{4 \pi} \operatorname{tr} \int d^{2} x\left(g_{n}^{-1} \star s_{+}^{(n)} \star g_{n} \star s_{-}^{(n)}-s_{+}^{(n)} \star s_{-}^{(n)}\right)\right],
\end{aligned}
$$

where the matrices $g_{i}$ are in the representation (2.11).

Differentiating (2.35) with respect to any of the components of the sources gives the correlation functions in the relevant chirality sector

$$
j_{+}^{(n)} \rightarrow \frac{i}{4 \pi} \operatorname{tr} g_{n}^{-1} \star \partial_{+} g_{n}, \quad j_{-}^{(n)} \rightarrow \frac{i}{4 \pi} \operatorname{tr} g_{n} \star \partial_{-} g_{n}^{-1}, \quad n=1,2 ;
$$

or in terms of the field components

$$
\begin{aligned}
& \bar{\psi} \star \gamma_{+} \psi \rightarrow \frac{i}{4 \pi} e_{\star}^{i \Lambda_{1}} \star \partial_{+} e_{\star}^{-i \Lambda_{1}}, \quad \bar{\psi} \star \gamma_{-} \psi \quad \rightarrow \quad \frac{i}{4 \pi} e_{\star}^{-i \Lambda_{1}} \star \partial_{-} e_{\star}^{i \Lambda_{1}} ; \\
& \psi_{\beta} \star\left(\gamma_{+}\right)_{\alpha \beta} \bar{\psi}_{\alpha} \rightarrow \frac{i}{4 \pi} e_{\star}^{-i \Lambda_{2}} \star \partial_{+} e_{\star}^{i \Lambda_{2}}, \quad \psi_{\beta} \star\left(\gamma_{-}\right)_{\alpha \beta} \bar{\psi}_{\alpha} \rightarrow \frac{i}{4 \pi} e_{\star}^{i \Lambda_{2}} \star \partial_{-} e_{\star}^{-i \Lambda_{2}} .
\end{aligned}
$$

Taking into account the representation (2.11) one can see that the global free fermion symmetries $U(1) \times U(1)$ of type (2.6) translate to the bosonized model (2.35) as

$$
g_{2} \rightarrow U_{2} g_{2}, \quad g_{1} \rightarrow U_{1}^{-1} g_{1}, \quad U_{2}=\operatorname{diag}\left(u_{2}, 1\right), \quad U_{1}=\operatorname{diag}\left(1, u_{1}\right)
$$

Then, considering the product $u_{2} u_{1}^{-1}\left(\alpha_{i}=\right.$ const.) as in $(2.7)$ for the global $U(1)$, the transformations (2.39) written for the product of field components becomes

$$
e_{\star}^{i \Lambda_{2}} \star e_{\star}^{i \Lambda_{1}} \rightarrow u_{2} u_{1}^{-1} e_{\star}^{i \Lambda_{2}} \star e_{\star}^{i \Lambda_{1}} .
$$

We consider the bosonic theory (2.35) in terms of the boson fields that appear naturally in the commutative limit corresponding to the field responsible for the global $U(1)$ charge and a decoupled free field, respectively (similar arguments have been used in [26] to study a gauge theory of a doubly $U(1)$ gauged matter in $\mathrm{NC}$ spacetime). So, in the $\theta \rightarrow 0$ the transformation (2.40) becomes

$$
e^{i \Lambda_{+}} \rightarrow u_{2} u_{1}^{-1} e^{i \Lambda_{+}}, \quad \Lambda_{ \pm} \equiv\left(\Lambda_{2} \pm \Lambda_{1}\right)
$$

Then, the charge corresponding to the global $U(1)$ symmetry of the $\mathrm{NC}$ free fermion should be related to the field $\Lambda_{+}$of the bosonic sector. Once the physical fields are identified, one can re-write the action of (2.35) in terms of them on NC spacetime. Thus, writing the action of (2.35) in terms of the fields $\Lambda_{ \pm}$one can see that these fields do not decouple from each other, whereas in commutative space they do. Let us discuss this point in more detail using the Polyakov-Wiegmnann identity (2.32). Since the last term in (2.32) vanishes for the representation (2.11), then the sum $W\left[g_{1}\right]+W\left[g_{2}\right]$ is equivalent to $W\left[g_{2} \star g_{1}\right]$ which 
couples the fields $\Lambda_{+}$and $\Lambda_{-}$in a non-trivial way. However, it can be seen that the fields $\Lambda_{-}$and $\Lambda_{+}$decouple in the commutative limit of the action (2.35). In this limit one has $W\left[g_{2} g_{1}\right]=\int d^{2} x \frac{1}{16 \pi}\left[\left(\partial_{\mu} \Lambda_{+}\right)^{2}+\left(\partial_{\mu} \Lambda_{-}\right)^{2}\right]$. Since the bosonized action of an ordinary free Dirac fermion in Minkowski spacetime corresponds just to one real scalar field, then one can set $\Lambda_{1}=\Lambda_{2}\left(\Lambda_{-}=0\right)$ in the commutative limit (however, see in the last paragraph of the next section a discussion regarding the complex character of the fields $\Lambda_{i}$ in the bosonization process). Moreover, in this limit the two currents differ only by a minus sign; in fact, by comparing (2.37) and (2.38) one has $j_{\mu}^{(1)}=-j_{\mu}^{(2)}$, as pointed out before. Regarding the discussions above, an important point is that the symmetry properties are simplest in terms of the fields $\Lambda_{1}$ and $\Lambda_{2}$ and the physical interpretation becomes direct when expressed in terms of the fields $\Lambda_{ \pm}$.

\section{The NC bi-fundamental massive Thirring model}

The (Euclidean) Lagrangian of the non-commutative massive Thirring model $\left(\mathrm{NCMT}_{1}\right)^{1}$ is

$$
\mathcal{L}_{N C M T}=i \bar{\psi} \gamma^{\mu} \partial_{\mu} \star \psi+m \bar{\psi} \star \psi-\frac{\lambda_{b f}}{4} j^{(1) \mu} \star j_{\mu}^{(1)}-\frac{\lambda_{b f}}{4} j^{(2) \mu} \star j_{\mu}^{(2)},
$$

defined for Dirac fields and with the currents given in (2.4)-(2.5). Here, $\lambda_{b f}$ is the relevant coupling constant and the group index contractions are being assumed.

The action related to (3.1) in terms of the spinor field components becomes

$$
\begin{aligned}
\mathcal{S}_{N C M T}= & \int d^{2} x\left[i \widetilde{\psi}_{L} \partial_{+} \psi_{L}+i \widetilde{\psi}_{R} \partial_{-} \psi_{R}+m\left(\widetilde{\psi}_{R} \psi_{L}+\widetilde{\psi}_{L} \psi_{R}\right)-\right. \\
& \left.\lambda_{b f}\left(\widetilde{\psi}_{R} \star \psi_{R} \star \widetilde{\psi}_{L} \star \psi_{L}+\psi_{R} \star \widetilde{\psi}_{R} \star \psi_{L} \star \widetilde{\psi}_{L}\right)\right],
\end{aligned}
$$

where again the group index notation has been suppressed for convenience.

Notice that the NCMT model (3.1) contains the non-standard interaction term $j_{\mu}^{(2)} \star$ $j^{(2) \mu} \sim \psi_{R} \star \widetilde{\psi}_{R} \star \psi_{L} \star \widetilde{\psi}_{L}$, which, to our knowledge, has not been considered previously in the literature. Since the currents $j^{(1) \mu}$ and $j^{(2) \mu}$ differ only by a sign in the commutative limit, the Lagrangian (3.1) reduces to the usual MT model in this limit. The bosonization process of the NC extension of the usual Thirring interaction performed in $[15,13]$ considers only $j_{\mu}^{(1)} \star j^{(1) \mu}$. The inclusion of a second $U(1)$ current will change various aspects of the bosonized model, such as the potential, the duality relationship and the field content, as we will show below.

The $U(1) \times U(1)$ symmetries give the associated conservation equations for the $\mathrm{NCMT}_{1}$ model

$$
\begin{aligned}
& \partial_{\mu} j^{(1) \mu}=0, \\
& \partial_{\mu} j^{(2) \mu}=0,
\end{aligned}
$$

where the currents $j_{\mu}^{(n)}(n=1,2)$ are of the type given in (2.4)-(2.5). The conservation laws (3.3)-(3.4) can be verified as the result of the field equations of motion [8]. As in the free

\footnotetext{
${ }^{1}$ Here we consider the fermionic analog of the (bosonic) model $\mathrm{NCMT}_{1}$ of Ref. [8].
} 
bi-fundamental fermion case the global $U(1)$ charge is associated to the transformation $u_{2} u_{1}^{-1}$ and the currents $j_{\mu}^{(1)}$ and $j_{\mu}^{(2)}$ share the same charge. In fact, the two currents are related by $\psi_{\alpha} \leftrightarrow \psi_{\alpha}^{\dagger}$. Therefore the Noether $U(1)$ charge is given by

$$
Q \equiv e_{n} \int d^{2} x j^{(n) 0}, \quad n=1 \text { or } 2
$$

where the $e_{n}$ are some normalization factors, which can be set $e_{1}=1, e_{2}=-1$.

\subsection{The Thirring type interactions and NC bosonization}

Let us bosonize the four-fermion interaction terms of the model (3.1), i.e.

$$
\mathcal{L}_{\lambda_{b f}}=-\frac{\lambda_{b f}}{4} j^{(1) \mu} \star j_{\mu}^{(1)}-\frac{\lambda_{b f}}{4} j^{(2) \mu} \star j_{\mu}^{(2)}
$$

Here we shall use directly the bosonization dictionary for the currents established in (2.37)-(2.38) ${ }^{2}$. Then, the four fermion interactions of (3.6) correspond to the following terms in the boson theory

$$
\begin{aligned}
-\frac{\lambda_{b f}}{4} \int d^{2} x 2\left(\bar{\psi} \star \gamma_{+} \psi\right) \star\left(\bar{\psi} \star \gamma_{-} \psi\right) & \rightarrow \frac{\lambda_{b f}}{32 \pi^{2}} \int d^{2} x \operatorname{tr} g_{1}^{-1} \star \partial_{+} g_{1} \star g_{1} \star \partial_{-} g_{1}^{-1}(3.7 \\
-\frac{\lambda_{b f}}{4} \int d^{2} x 2\left(\psi_{\beta} \star\left(\gamma_{+}\right)_{\alpha \beta} \bar{\psi}_{\alpha}\right) \star\left(\psi_{\sigma} \star\left(\gamma_{-}\right)_{\delta \sigma} \bar{\psi}_{\delta}\right) & \rightarrow \frac{\lambda_{b f}}{32 \pi^{2}} \int d^{2} x \operatorname{tr} g_{2}^{-1} \star \partial_{+} g_{2} \star g_{2} \star \partial_{-} g_{2}^{-1} .(3.8
\end{aligned}
$$

It is known that the bosonized four fermion interaction term in the commutative case adds to the kinetic term an extra contribution, whereas the right hand side terms in (3.7)-(3.8) contribute an infinite series of theta dependent higher derivative terms to the first quadratic term. In this way the $\mathrm{NC}$ extension provides additional terms making the bosonized theory in fact very different from the commutative version.

The bosonized Lagrangian for the kinetic and four-fermion interaction terms becomes

$$
\begin{array}{r}
W\left[g_{1}\right]+W\left[g_{2}\right]+\frac{\lambda_{b f}}{32 \pi^{2}} \int d^{2} x \operatorname{tr} g_{2}^{-1} \star \partial_{+} g_{2} \star g_{2} \star \partial_{-} g_{2}^{-1}+ \\
\frac{\lambda_{b f}}{32 \pi^{2}} \int d^{2} x \operatorname{tr} g_{1}^{-1} \star \partial_{+} g_{1} \star g_{1} \star \partial_{-} g_{1}^{-1}
\end{array}
$$

In order to understand better the bosonic sector we need to uncover a canonically normalized scalar field related to the global $U(1)$ symmetry which corresponds to the charge of the fermion field. So, let us consider the scalar fields $\Lambda_{i}$ defined in (2.11). Then, the $\theta$ expansion of (3.9) provides

$$
\frac{1}{4 \pi} \sum_{i=1}^{2} \int d^{2} x \partial_{+} \Lambda_{i} \partial_{-} \Lambda_{i}+\frac{\lambda_{b f}}{32 \pi^{2}} \sum_{i=1}^{2} \int d^{2} x \partial_{+} \Lambda_{i} \partial_{-} \Lambda_{i}+\ldots
$$

\footnotetext{
${ }^{2}$ There exists another prescription to bosonize the current-current coupling using the "completing the square" type Hubbard-Stratonovich identity which also holds in the NC case [15].
} 
Taking into account these contributions and the definitions (2.41) one gets

$$
\frac{1}{8 \pi}\left(1+\frac{\lambda_{b f}}{8 \pi}\right) \int d^{2} x\left[\partial_{+} \Lambda_{+} \partial_{-} \Lambda_{+}+\partial_{+} \Lambda_{-} \partial_{-} \Lambda_{-}\right]+\ldots,
$$

therefore, the canonically normalized scalar related to the fermion charge corresponds to

$$
\Phi_{+} \equiv\left[\frac{1}{8 \pi}\left(1+\frac{\lambda_{b f}}{8 \pi}\right)\right]^{1 / 2} \Lambda_{+} .
$$

This result will be used below to relate the two-field NC sine-Gordon and $\mathrm{NCMT}_{1}$ coupling constants. Observe that for stability of the bosonic theory we must have $\lambda_{b f}>-8 \pi$. The allowed range of the coupling gets enlarged as compared to the anti-fundamental massless Thirring which is $\lambda_{f}>-4 \pi$ [13]. Recall that for the ordinary massless Thirring model this bound is $\lambda>-\pi[9]$. Thus, there is a whole range of coupling constants, depending on the fermion representation, for which the NC massless Thirring is a sensible model.

At this stage we have reproduced two copies of the WZNW action associated, respectively, to the fields $\Lambda_{1}$ and $\Lambda_{2}$, and additional terms in (3.9) due to the current-current couplings. A two-field potential will emerge from the bi-fundamental fermion mass term, as we will see in the next subsection.

\subsection{The mass term and NC bosonization}

Next we consider the bosonization of the bi-fundamental fermion mass term. The relevant term is

$$
S_{M}=m \int d^{2} x\left(\psi_{R i_{1}}^{\dagger j_{1}} \star \psi_{L j_{1}}^{i_{1}}+\psi_{L}^{\dagger j_{1}} \star \psi_{R j_{1}}^{i_{1}}\right)
$$

Here, we follow the procedure implemented in Refs. $[17,13]$ in which the chiral symmetry plays a central role in the bosonization process. In our case this procedure still applies since the free bi-fundamental fermion possesses a global axial symmetry $U_{5}(1) \times U_{5}(1)$ for which the starred products collapse to the ordinary ones. In fact, the free fermion theory (2.2) is symmetric under the global chiral symmetry

$$
(\psi)^{T} \rightarrow\left(e^{i \alpha_{2} \gamma_{5}} \psi\right)^{T} e^{i \alpha_{1} \gamma_{5}} ; \quad(\bar{\psi})^{T} \rightarrow\left(e^{i \alpha_{1} \gamma_{5}} \bar{\psi}\right)^{T} e^{i \alpha_{2} \gamma_{5}},
$$

where $T$ stands for matrix transpose operation and the suggestive products have been written in spite of their global character. It is assumed that the bosonic theory preserves this symmetry [20]. Besides, the duality approach to bosonization relies on gauging the vector $U(1) \times U(1)$ symmetries $[17,18]$. On the other hand, it is a known fact that the axial symmetry does not survive quantization. The relevant axial anomalies in $\mathrm{NC}$ plane are $[14,15,28,31]$

$$
\begin{aligned}
& \partial_{\mu} j_{5}^{(2) \mu}=\frac{1}{2 \pi} \epsilon^{\mu \nu} F_{\mu \nu}^{(2)}, j_{5}^{(2) \mu}=\psi_{\beta} \star\left(\gamma^{\mu} \gamma_{5}\right)_{\alpha \beta} \bar{\psi}_{\alpha}, \\
& \partial_{\mu} j_{5}^{(1) \mu}=-\frac{1}{2 \pi} \epsilon^{\mu \nu} F_{\mu \nu}^{(1)}, \quad j_{5}^{(1) \mu}=\bar{\psi} \star \gamma^{\mu} \gamma_{5} \psi,
\end{aligned}
$$


where the $F_{\mu \nu}^{(i)}$ are the NC gauge field strengths.

In order to make the axial symmetry manifest during the bosonization process the authors of [17] provided the lagrange multiplier with a transformation property to cancel the fermion axial anomaly. In fact, the gauged action would have the terms

$$
\sim \exp \left(\int d^{2} x\left[\frac{1}{2 \pi} \Lambda_{1} \star \epsilon^{\mu \nu} F_{\mu \nu}^{(1)}-\frac{1}{2 \pi} \Lambda_{2} \star \epsilon^{\mu \nu} F_{\mu \nu}^{(2)}\right]\right)
$$

The key point is that if the fields $\Lambda_{i}$ transform as $\Lambda_{i} \rightarrow \Lambda_{i}-\alpha_{i}$ under axial transformations, then the Lagrange multiplier terms (3.17) will cancel the axial anomaly terms (3.15)-(3.16) which emerge in the path-integral, thus making the whole generating functional manifestly chiral symmetric. These considerations tell us how the $\Lambda_{i}$ fields transform under chiral rotations [17].

Even though the mass term is no longer axial symmetric, these transformation properties allow us to bosonize the mass term. The observation is that these transformation rules remain the same for the bilinear terms in (3.13). The problem resides in finding the bosonic functionals associated to these bilinear terms, $\mathcal{F}\left(\Lambda_{1}, \Lambda_{2}\right) \equiv \psi_{R i_{1}}^{\dagger j_{1}} \star \psi_{L j_{1}}^{i_{1}}$ and its corresponding $\mathcal{F}^{\dagger}\left(\Lambda_{1}, \Lambda_{2}\right)$. Here the functionals depend on $\Lambda_{i}$ through $\star$-products. Under the global symmetry transformations (3.14) one has

$$
\psi_{R i_{1}}^{\dagger j_{1}} \star \psi_{L j_{1}}^{i_{1}} \rightarrow e^{-2 i\left(\alpha_{1}+\alpha_{2}\right)} \psi_{R j_{1}}^{\dagger i_{1}} \star \psi_{L j_{1}}^{i_{1}}
$$

and taking into account the $\Lambda_{i}$ field transformations discussed above we have the functional transformation property

$$
\mathcal{F}\left(\Lambda_{1}-\alpha_{1}, \Lambda_{2}-\alpha_{2}\right)=e^{-2 i\left(\alpha_{1}+\alpha_{2}\right)} \mathcal{F}\left(\Lambda_{1}, \Lambda_{2}\right) .
$$

Therefore, one has that

$$
\mathcal{F}\left(\Lambda_{1}, \Lambda_{2}\right) \sim e_{\star}^{2 i \Lambda_{1}} \star e_{\star}^{2 i \Lambda_{2}}
$$

uniquely solves (3.19) for globally defined rotations. With this result the mass term (3.13) becomes

$$
S_{M}=\int d^{2} x m \alpha_{0}\left(e_{\star}^{2 i \Lambda_{1}} \star e_{\star}^{2 i \Lambda_{2}}+e_{\star}^{-2 i \Lambda_{2}} \star e_{\star}^{-2 i \Lambda_{1}}\right),
$$

where $\alpha_{0}$ is a parameter to be associated to the zero point energy.

This is precisely the potential proposed by Lechtenfel et al. for the NC sine-Gordon [4]. In the previous section we associated the field $\Lambda_{+}=\left(\Lambda_{1}+\Lambda_{2}\right)$ to the charged sector of the bi-fundamental NC free fermion model. It is expected that the same field will be associated to the global $U(1)$ charge of the $\mathrm{NCMT}_{1}$ theory as discussed in the paragraph after the Eqs. (3.3)-(3.4). This field has been isolated from the quadratic and quartic terms in (3.11). So, we would like to uncover this field in the bosonized mass term (3.21) as well. Actually, this is not possible to do exactly due to the $\star$-product involved and only one can pursue it in the $\theta$ perturbation expansion. Then the expansion of the potential provides

$$
\int d^{2} x m \alpha_{0}\left[2 \cos \left(2 \Lambda_{+}\right)+\ldots\right] .
$$


The canonically normalized field $\Phi_{+}$was defined in $(3.12)$, then the first potential term becomes

$$
\int d^{2} x m \alpha_{0}\left[2 \cos \left(\beta_{b f} \Phi_{+}\right)+\ldots\right]
$$

where

$$
\frac{32 \pi}{\beta_{b f}^{2}}=1+\frac{\lambda_{b f}}{8 \pi}
$$

Thus, we have a S-duality relationship. The relation (3.24) constitutes the quantum version of the strong-weak duality obtained in the classical 1 -soliton sector of the $\mathrm{NCMT}_{1}$ $\rightarrow \mathrm{NCSG}_{1}$ correspondence [8].

This type of relationship has also been derived in [13] for the NC bosonization of the massive Thirring model with anti-fundamental fermion (see eq. (1.4)). With these results in hand one can establish that the couplings of the various models are related by

$$
\lambda_{b f}=2 \lambda_{\bar{f}}=8 \lambda_{M T}, \quad \beta_{b f}^{2}=2 \beta_{\bar{f}}^{2}=8 \beta_{S G}^{2},
$$

where the $\lambda_{\bar{f}}, \beta_{\bar{f}}^{2}$ couplings correspond to the fermionic and bosonic sector considered in [13], respectively ${ }^{3}$. The couplings $\lambda_{M T}, \beta_{S G}^{2}$ were defined in (1.1)-(1.2) for the models in ordinary spacetime.

Notice that the duality relation is maintained in each term of the $\theta$ expansions of the potential terms (3.21), as well as the quadratic and quartic terms of (3.9). To see this fact notice that in order to define the canonically normalized field $\Phi_{+}$the fields $\Lambda_{i}$ have been re-scaled as $\Lambda_{i} \rightarrow \frac{\beta}{2} \Lambda_{i}$, then the expansions will only produce terms proportional to positive powers of the bosonic coupling constant $\beta$.

In the bosonic sector we must have a topological charge corresponding to the global $U(1)$ Noether charge of the fermionic theory. The physical scalar field associated to this topological charge may be identified in the commutative limit $\theta \rightarrow 0$ of the model. The field $\Lambda_{-}$decouples completely in this limit since the quartic terms contributions are only of the bilinear kinetic type and the combination $\Lambda_{+}$alone appears in the potential term. So, it is clear that the field $\Phi_{+}$defined in (3.12) carries this charge in the bosonic sector. Then, we can define $[5,8]^{4}$

$$
j_{\text {top }}^{\mu} \equiv \frac{\beta_{b f}}{2 \pi} \epsilon^{\mu \nu} \partial_{\nu} \Phi_{+}, \quad Q_{\text {top }}=\frac{\beta_{b f}}{2 \pi} \int_{-\infty}^{\infty} d x^{1} \frac{d \Phi_{+}}{d x^{1}} \equiv \sum_{n=0} \theta^{n} Q_{n},
$$

where $Q_{t o p}$ is the topological charge expressed as a power series in $\theta$. This expression is the same as in the classical $\mathrm{NCSG}_{1}$ model. In fact, for the classical one (anti-)soliton sector it has

\footnotetext{
${ }^{3}$ It is expected that by formally setting $\lambda_{f} \equiv \lambda_{\bar{f}}, \beta_{f}^{2} \equiv \beta_{\bar{f}}^{2}$ in (1.4) one can obtain the same duality relationship for fundamental fermion.

${ }^{4}$ The bosonization rules (2.37)-(2.38) and the Noether charge definition (3.5) might suggest for $j_{\text {top }}^{\mu}$ to be some combinations of the type $e_{\star}^{ \pm i \Lambda_{j}} \star \partial_{ \pm} e_{\star}^{\mp i \Lambda_{j}},(j=1,2)$. However, it is a fact that only the field $\Phi_{+}\left(\sim \Lambda_{+}\right)$ appears in the commutative limit of the potential and since the $Q_{\text {top }}$ is related to a discrete symmetry of the potential it is plausible to define it as in (3.26).
} 
been obtained an exact topological charge equal to \pm 1 , respectively; i.e. one has in (3.26), $Q_{n}=0(n>0)$, since the 1 -soliton solution of the ordinary sine-Gordon model solves also the NC theory $[4,8,32]$. The Noether and topological currents equivalence is also present in the ordinary MT/SG relationship, on the quantum $[9,16]$ and classical level establishing a soliton/particle correspondence [10,33].

Finally, the $\mathrm{NCMT}_{1}$ model (3.1) is equivalent to a bosonic model which comprises the eq. (3.9) plus the eq. (3.21), which we record below in matrix notation

$$
\begin{aligned}
\mathcal{S}_{b f}\left[g_{1}, g_{2}\right]= & W\left[g_{1}\right]+W\left[g_{2}\right]+\int d^{2} x \operatorname{tr}\left[m \alpha_{0}\left(\sigma_{1} g_{2}^{-2} \star g_{1}^{-2} \sigma_{1} \star g_{1}^{2} \star g_{2}^{2}\right)+\right. \\
& \left.\frac{\lambda_{b f}}{32 \pi^{2}}\left(g_{2}^{-1} \star \partial_{+} g_{2} \star g_{2} \star \partial_{-} g_{2}^{-1}+g_{1}^{-1} \star \partial_{+} g_{1} \star g_{1} \star \partial_{-} g_{1}^{-1}\right)\right],
\end{aligned}
$$

where the matrices $g_{1}, g_{2}$ are given in $(2.11)$ and $\sigma_{1}=\left[\begin{array}{ll}0 & 1 \\ 1 & 0\end{array}\right]$. Thus, the bosonic sector comprises two WZW actions plus a potential depending on two scalar fields (thus, the first three terms of (3.27) correspond to the Lechtenfeld et al. proposal [4] for NC sine-Gordon) and additional quartic terms coming from the current-current interactions. These quartic terms were also absent in the $\mathrm{NCSG}_{1}$ model in the purely classical treatments of [8].

The upper and lower indices of a model defined for bi-fundamental matter can be interchanged since the metric is $\delta_{A B}$ in the normalization $\operatorname{tr}\left(T_{A} T_{B}\right)=\frac{1}{2} \delta_{A B}$ of the Lie algebra generators. This symmetry can be written manifestly using the group index notation as in (2.1) or (3.13) for the $\mathrm{NCMT}_{1}$ model (3.1). Notice that the symmetry $i \leftrightarrow j$ which interchanges the two fundamental representations corresponds to the symmetry $g_{1} \leftrightarrow g_{2}$ of the bosonic sector (3.27) (recall that $\left.g_{1} \star g_{2}=g_{2} \star g_{1}\right)$. The action (3.27) can be written in a more compact form using the Polyakov-Wiegmann identity and the representation (2.11) for $g \in U(1) \times U(1)$

$$
\mathcal{S}_{b f}[g]=W[g]+\int d^{2} x \operatorname{tr}\left[m \alpha_{0}\left(\sigma_{1} g^{-2} \sigma_{1} \star g^{2}\right)+\frac{\lambda_{b f}}{32 \pi^{2}}\left(g^{-1} \star \partial_{+} g \star g \star \partial_{-} g^{-1}\right)\right] .(3
$$

This representation is more convenient in order to compare it to its analog model obtained from reduction of the parent action, the $G L(2, C) \mathrm{NC}$ affine Toda model coupled to matter (Dirac) fields [8]. Except for the last quartic term, this is the $\mathrm{NCSG}_{1}$ model defined in [8, 4]. Recall that the theories $\mathrm{NCSG}_{1,2}$ defined in [8] correspond to the Lechtenfeld et al. and Grisaru-Penati models, respectively. The Grisaru-Penati model has the potential $\left(e_{\star}^{i \phi}+e_{\star}^{-i \phi}\right)$ corresponding to one complex scalar field, instead of the potential (3.21) defined for two fields of the Lechtenfeld et al. model. Strictly speaking, in our constructions above the fields $g_{i} \in U(1)_{C}$ (complexified $U(1)$ ), because the determinants were computed in Euclidean space. This is the case in ordinary commutative space, in which the complexification of $U(1), U(1)_{C}$ is considered [34]. In the construction of the model $\mathrm{NCSG}_{1}$ we may consider the abelian subgroup of $G L(2, C), U(1)_{C} \times U(1)_{C}$ to which the fields $g_{1}$ and $g_{1}$ belong, respectively [8]. Nevertheless, it is a fact that the $\mathrm{NCSG}_{1}$ model possesses real soliton type solutions for the field $\Lambda_{+}\left(=\Lambda_{1}+\Lambda_{2}\right)$, whereas $\Lambda_{-}\left(=\Lambda_{1}-\Lambda_{2}\right)$ remains as a decoupled free field $[8,4]$. 


\section{Conclusions and discussions}

The NC bosonization of the $\mathrm{NCMT}_{1}$ model and some properties emerging from this process have been considered. In order to bosonize the current-current interactions we have used the bosonization dictionary (2.36) established for the relevant currents of a bi-fundamental free fermion. In order to bosonize the mass term we have used the axial transformation rules that the fields $\Lambda_{i}$ must undergo in order to maintain these symmetries manifest during the bosonization process of a free fermion as developed in NC space [13], following the ordinary space approach of [17]. Even though the mass term breaks the both axial symmetries the axial transformation rules remain the same, thus allowing to construct the boson sector of the fermion mass term. The bosonized mass term reproduces the Lechtenfeld et al. proposal for the NC sine-Gordon potential [4,8]. It is uncovered the duality relationship between the coupling constants of the both sectors (3.24), as well as certain relationships between the couplings for the models with bi-fundamental, (anti-)fundamental fermion representations and their bosonic sectors and the ordinary MT/SG couplings (3.25). The global $U(1)$ charge of the fermionic sector (3.5) defined for either, the current $j^{(1) \mu}$ or $j^{(2) \mu}$, corresponds to the topological charge (3.26) defined for the field $\Phi_{+}\left(\sim \Lambda_{+}\right)$. It is shown that the auxiliary field $\Lambda_{-}$decouples in the commutative limit. However the deformed situation requires the both scalar fields $\Lambda_{ \pm}$.

Disregarding for the moment the quantum nature of the terms, such as certain normal ordering prescriptions, the bosonic (3.27) [or (3.28)] and fermionic (3.1) models resemble to their classical counterparts $\mathrm{NCSG}_{1}$ and $\mathrm{NCMT}_{1}$, respectively, as defined in [8] (except for the last quartic term in (3.28). A remarkable feature of the $\mathrm{NCSG}_{1}$ model, as well as some $\mathrm{NC}$ integrable systems [32], is that for $1-$ (anti)soliton type solution the $\star$-products in its equations of motion collapse to ordinary ones since one has in general $f(x-v t) \star g(x-v t)=$ $f(x-v t) g(x-v t)$. Then the $\star$-products for these type of fields in the equations of motion of the model (3.28) reduce to the ordinary ones. In particular, the contributions from the last quartic term in (3.28) are only of the type emerging from the kinetic terms of the action. So, we may say that regarding the classical 1-(anti)soliton type solution, the bosonized model (3.28) and the classical $\mathrm{NCSG}_{1}$ theories are very similar. This is in contrast to the usual $\mathrm{MT} / \mathrm{SG}$ duality in which the corresponding classical and quantum Lagrangians look very similar, apart from the field renormalizations and the relevant quantum corrections to the coupling constants.

Various aspects of the bosonized model derived above deserve attention in future research, e.g. the properties of the S-matrix, the $\mathrm{NC}$ zero-curvature formulation and integrability properties, the NC multi-soliton spectrum and their scattering properties. The ordinary equivalence $\mathrm{SG} / \mathrm{MT}$ has been used to understand some confinement mechanism in $\mathrm{QCD}_{2}$ with one flavor and $\mathrm{N}$ colors [35], on this regard it would be interesting to consider the NC situation. Moreover, the multi-fermion extensions of the $\mathrm{NCMT}_{1}$ type model also deserve attention, e.g. in connection to multi-flavor noncommutative $\mathrm{QCD}_{2}$. In ordinary space the correspondence between the multi-fermion massive Thirring and multi-boson sine-Gordon models related to any (untwisted) affine Lie algebra has been considered in [12] on the classical level. The classical correspondence between three-fermion massive Thirring and three-boson sine-Gordon has been addressed in [36] and the quantum field theory aspects 
studied in [37] through bosonization techniques. It would be interesting to address their NC extensions.

\section{Acknowledgments}

The author thanks H.L. Carrion and M. Rojas for collaboration in a previous work and to ICET and Prof. M. C. Araújo at the Mathematics Department-UFMT for hospitality. This work has been supported by CNPq-FAPEMAT.

\section{References}

[1] N. Seiberg and E. Witten, JHEP 9909 (1999) 032;

A. Connes, M. R. Douglas and A. Schwarz, JHEP 9802 (1998) 003;

M. R. Douglas and C. M. Hull, JHEP 9802 (1998) 008.

[2] O. Lechtenfeld, A. D. Popov and B. Spendig, Phys. Lett. 507B (2001) 317.

[3] K. Takasaki, J. Geom. Phys. 37 (2001) 291.

[4] O. Lechtenfeld, L. Mazzanti, S. Penati, A. D. Popov, L. Tamassia, Nucl. Phys. B705 (2005) 477.

[5] M. T. Grisaru and S. Penati, Nucl. Phys. B655 (2003) 250.

[6] M.T. Grisaru, L. Mazzanti, S. Penati and L.Tamassia, JHEP 0404 (2004) 057.

[7] I. Cabrera-Carnero and M. Moriconi, Nucl. Phys. B673 (2003) 437.

[8] H. Blas, H.L. Carrion and M. Rojas, JHEP 0503 (2005) 037; hep-th/0502051.

[9] S. Coleman, Phys. Rev. D11 (1975) 2088;

S. Mandelstam, Phys. Rev. D11 (1975) 3026.

[10] H. Blas, Nucl. Phys. B596 (2001) 471;

H. Blas and B.M. Pimentel, Annals Phys. 282 (2000) 67.

[11] L.A. Ferreira, J.L. Gervais, J. Sanchez Guillen and M.V. Savelev, Nucl. Phys. B470 (1996) 236.

[12] H. Blas, JHEP 0311 (2003) 054.

[13] C. Nunez, K. Olsen and R. Schiappa, JHEP 0007 (2000) 030.

[14] E. F. Moreno and F. A. Schaposnik, JHEP 0003 (2000) 032.

[15] E. F. Moreno and F. A. Schaposnik, Nucl. Phys. B596 (2001) 439.

[16] C. M. Naon, Phys. Rev. D31 (1985) 2035. 
[17] C. P. Burgess and F. Quevedo, Nucl. Phys. B421 (1994) 373.

[18] C. P. Burgess and F. Quevedo, Phys. Lett. 329B (1994) 457.

[19] J. C. Le Guillou, E. Moreno, C. Nunez and F. A. Schaposnik, Nucl. Phys. B484 (1997) 682.

[20] E. Witten, Commun. Math. Phys. 92 (1984) 455.

[21] O. J. Ganor, G. Rajesh and S. Sethi, Phys. Rev. D62 (2000) 125008;

R. Gopakumar, J. M. Maldacena, S. Minwalla and A. Strominger, JHEP 0006 (2000) 036 ;

J. X. Lu, S. Roy and H. Singh, JHEP 0009 (2000) 020;

R. G. Cai and N. Ohta, Prog. Theor. Phys. 104 (2000) 1073; JHEP 0003 (2000) 009;

R.G. Cai, J.-X. Lu and N. Ohta, Phys. Lett. 551B (2003) 178.

[22] N. Seiberg, L. Susskind and N. Toumbas, JHEP 0006 (2000) 044.

J. Gomis, T. Mehen, Nucl. Phys. B591 (2000) 265.

[23] C-S. Chu, J. Lukierski, W.J. Zakrzewski, Nucl. Phys. B632 (2002) 219.

[24] K. Furuta and T. Inami, Mod. Phys. Lett. A15 (2000) 997.

[25] J. M. Gracia-Bondia and C. P. Martin, Phys. Lett. 479B (2000) 321.

[26] Y. Liao and K. Sibold, Phys. Lett. 586B (2004) 420.

[27] S. Terashima, Phys. Lett. 482B (2000) 276.

[28] T. Nakajima, Phys. Rev. D68 (2003) 065014.

[29] L. Bonora and A. Sorin, Phys. Lett. 521B (2001) 421.

[30] C. P. Martin, Nucl. Phys. B623 (2002) 150.

[31] F. Ardalan and N. Sadooghi, Int. J. Mod. Phys. A16 (2001) 3151; Int. J. Mod. Phys. A17 (2002) 123.

[32] A. Dimakis and F. Mueller-Hoissen, A noncommutative version of the nonlinear schroedinger equation, hep-th/0007015.

[33] S. J. Orfanidis, Phys. Rev. D14 (1976) 472;

S. J. Orfanidis and R. Wang, Phys. Lett. 57B (1975) 281.

[34] S. G. Naculich and H. J. Schnitzer, Nucl. Phys. B332 (1990) 583;

R. E. Gamboa Saravi, F. A. Schaposnik and J. E. Solomin, Phys. Rev. D30 (1984) 1353.

[35] H. Blas and L.A. Ferreira, Nucl. Phys. B571 (2000) 607;

H. Blas, Phys. Rev. D66 (2002) 127701. 
[36] J. Acosta, H. Blas, J. Math. Phys. 43 (2002) 1916, see also hep-th/0407020;

[37] H. Blas, Eur. Phys. J. C37 (2004) 251, see also hep-th/0409269. 